J. Korean Math. Soc. 43 (2006), No. 6, pp. 1289-1300

\title{
ANTI-HOLOMORPHIC TWISTOR AND SYMPLECTIC STRUCTURE
}

\author{
DOSANG JOE
}

\begin{abstract}
ABSTRA:T. It is well known that the twistor, section of twistor space, classify the orthogonal almost complex structure on even dimensional Riemannian manifold $(X, g)$. We will show that existence of a harmonic and anti-holomorphic twistor is equivalent to having a symplectic structure on $(X, g)$.
\end{abstract}

\section{Introduction}

By definition, a manifold having a non-degenerate closed two form $\omega$ is called a syr.plectic manifold. This category of manifolds was firstly understood as that of Kähler manifolds, which has even odd betti number, for example, later on some mathematician like B. Thurston and R. Gompf constructed examples of symplectic manifolds which cannot have Kähler structure. Moreover R. Gompf [2] find a systematic way of constructing symplectic manifolds and show that every finitely presented group can be realized as a fundamental group of a symplectic 4-manifold. It reveals that the symplectic category is much more bigger than Kähler one and expected to be characterized as cohomology condition of given manifold such as $a \in H^{2}(X, \mathbf{R})$ and $0 \neq a \cup \cdots \cup a \in H^{2 m}(X, \mathbf{R})$. This expectation has been broken in the advent of Seiberg-Witten theory for the 4-dimensional topology. It has been known that every symplectic 4manifold has non-zero Seiberg-Witten invariants $[5,6]$, which indicates that condition of having symplectic structure on 4-manifolds is quite subtle. Taking closer look at the Taubes's paper [5], we can find that he was making use of the characterization of symplectic form, which is there are canonical $\mathrm{Spin}^{c}$ structure associated almost complex structure $J$ and naturally induced a nowhere vanishing positive spinor $u$ which is harmonic, i.e., $\mathbb{D} u=0$. In this paper, we are going to show that such a

Received July 17, 2005.

2000 Mathemetics Subject Classification: 53D05, 53C28.

Key words and phrases: twistor space, pure spinor, symplectic structure. 
characterization is equivalent to the existence of the symplectic form on a given manifold. First of all, note that symplectic form $\omega$ on a given manifold realized as an imaginary part of Hermitian metric for some almost complex structure $J$ on $T X$. Hence the existence of almost complex structure is a necessary condition for that of symplectic structure. Given a Riemannian even dimensional manifold $(X, g)$, the orthogonal almost complex structure is equivalent to a section of the twistor space which is a canonical fiber bundle of $S O(2 m) / U(m)$. We will discuss on this in the section 2. After choosing a twistor $u$, equivalently having an almost complex structure $J$, there is the naturally associated $\operatorname{Spin}_{2 m}$ equivariant Hermitian metric on $(T X, g, J)$ and a canonical $\operatorname{Spin}^{C}$ representation. The imaginary part of the Hermitian metric $\omega$ is our candidate for the symplectic form. It can be proved that the condition for $d \omega=0$ is equivalent to the section $u$ is anti-holomorphic and harmonic $(\mathbb{D} u=0)$, where $u$ can be understood as a nowhere-vanishing positive spinor of the canonical positive spinor bundle. To prove this theorem is the main purpose of this paper. It also gives a simple characterization of symplectic structure on smooth 4-manifolds, which is the same as the Taubes' analysis of symplectic form. Conclusively, the condition for $\omega$ being a symplectic form is $m(m-1) / 2+m(m-1)(m-2) / 6$ which is stronger than that condition for being integrable which is $m(m-1) / 2$, in the sense of the number of equation required. This is just because the we study the symplectic structure as an almost complex structure compatible with a given metric $g$. It suggests that there should be more restrictive twistor, i.e., almost complex structure compatible with given metric $g$ which supports symplectic structures on a manifold. Let us introduce the preliminaries about the pure spinor and twistor.

\section{Pure spinor and twistor}

The following materials are collected from the book of Lawson and Michesohn [3]. Fix $\mathbf{R}^{n}$ be the standard inner product $(\langle\rangle$,$) real vector$ space and extend this metric C-linearly to $\mathbf{C}^{n}=R^{n} \otimes \mathbf{C}$. Let $\mathbf{C l}_{n}=$ $C l_{n} \otimes \mathbf{C}$ be the associated complexified Clifford algebra. Let $\mathbf{S}_{\mathbf{C}}$ be the fundamental $\mathbf{C l}_{n}$-module which defines the irreducible complex spinor space. For each spinor $\sigma \in \mathbf{S}_{\mathbf{C}}$, we can consider the $\mathbf{C}$-linear map

$$
j_{\sigma}: \mathbf{C}^{n} \rightarrow \boldsymbol{S}_{\mathbf{C}} \quad \text { given by } j_{\sigma}(v) \equiv v \cdot \sigma .
$$

Generically, this map is injective. However, there are interesting spinors for which $\operatorname{dim}\left(\operatorname{ker} j_{\sigma}\right)>0$. 
Definition 2.1. A complex subspace $V \subset \mathbf{C}^{n}$ is said to be isotropic (with respect to the bilinear form $\langle\cdot, \cdot\rangle$ ) if $\langle v, w\rangle=0$ for all $v, w \in V$.

We define a hermitian inner product $(\cdot, \cdot)$ on $\mathbf{C}^{n}$ by setting $(v, w)=$ $\langle v, \bar{w}\rangle$. Clearly, if $V \subset \mathbf{C}^{n}$ is an isotropic subspace, then $V \perp \bar{V}$ in this hermitian inner product. In particular, therefore, we have

$$
2 \operatorname{dim}_{\mathbf{C}} V \leq n \text {. }
$$

Definition 2.2. A spinor $\sigma$ is pure if $\operatorname{ker} j_{\sigma}$ is a maximal isotropic subspace, i.e., if $\operatorname{dim}\left(\operatorname{ker} j_{\sigma}\right)=[\mathrm{n} / 2]$.

Denote by $P \$$ the subset of pure spinors in $\$_{C}$, and denote by $\mathcal{I}_{n}$ the set of maximal isotropic subspaces of $\mathbf{C}^{n}$ Both $P \boldsymbol{\$}$ and $I_{n}$ are naturally acted upon by the group $\operatorname{Pin}_{n}$, and the assignment $\sigma \mapsto \operatorname{ker} j_{\sigma}$ gives a $\operatorname{Pin}_{n}$-equivariant map

$$
K: P \boldsymbol{S} \longmapsto \mathcal{I}_{n} .
$$

From this poirit on we shall assume that $n=2 m$ is an even integer, and furthermore that $\mathbf{R}^{2 m}$ is oriented.

Definition 2.3. An orthogonal almost complex structure on $\mathbf{R}^{2 m}$ is an orthogonal transformation $J: \mathbf{R}^{2 m} \rightarrow R^{2 m}$ which satisfies $J^{2}=$ -Id. For any such $J$, an associated unitary basis of $\mathbf{R}^{2 m}$ is an ordered orthonormal basis of the form $\left\{e_{1}, J e_{1}, \ldots, e_{m}, J e_{m}\right\}$. Any two unitary bases for a given $J$ determine the same orientation. This is called the canonical orientation associated $J$.

Let $\mathcal{C}_{m}$ denote the set of all orthogonal almost complex structures on $\mathbf{R}^{2 m}$. It is easy to see that $\mathcal{C}_{m}$ is a homogeneous space for the group $\mathrm{O}_{2 m}$. It falls into two connected components $\mathcal{C}_{m}^{+}$and $\mathcal{C}_{m}^{-}$, where $\mathcal{C}_{m}^{+} \cong$ $S O_{2 m} / U_{m}$ consists of those almost complex structures whose canonical is positive (i.e., agrees with given one on $\mathbf{R}^{2 m}$ ). Associated to any $J \in \mathcal{C}_{m}$ there is a decomposition

$$
\mathbf{C}^{2 m}=V(J) \oplus \overline{V(J)},
$$

where

$$
V(J) \equiv\left\{v \in \mathbf{C}^{2 m}: J v=-i v\right\}=\left\{v_{0}+i J v_{0}: v_{0} \in \mathbf{R}^{2 m}\right\} .
$$

There is an $O_{2 m}$-equivalent bijection

$$
\mathcal{C}_{m} \stackrel{V}{\longrightarrow} \mathcal{I}_{2 m}
$$

which associates to $J$ the isotropic subspace $V(J)$ Let $\mathcal{I}_{2 m}^{+}$denote the component corresponding to $\mathcal{C}_{m}^{+}$. Using the complex volume element $\omega_{\mathbf{C}}=i^{m} e_{1} \cdots e_{2 m}$, we have a decomposition $\boldsymbol{S}_{\mathbf{C}}=\mathbf{S}_{\mathbf{C}}^{+} \oplus \boldsymbol{S}_{\mathbf{C}}^{-}$into +1 
and -1 eigenspace respectively. Easy calculation gives a decomposition $P \boldsymbol{S}=P \mathbf{S}^{+} \coprod P \mathbf{S}^{-}$of the pure spinor space into positive and negative types. Let $\mathbf{P}\left(P \boldsymbol{S}^{+}\right)$denote the projectivization of the pure spinor space, i.e., $\mathbf{P}\left(P \mathbf{S}^{+}\right)=P \$ / \sim$, where we say that $\sigma \sim \sigma^{\prime}$ if $\sigma=t \sigma^{\prime}$ for some $t \in \mathbf{C}$. Each of the space $\mathbf{P}\left(P \boldsymbol{\beta}^{ \pm}\right), \mathcal{C}_{m}^{ \pm}$and $\mathcal{I}_{2 m}^{ \pm}$are acted upon by $\mathrm{Spin}_{2 m}$, in fact by $\mathrm{SO}_{2 m}$.

Proposition 2.4. The maps $\sigma \mapsto K(\sigma)$ and $J \mapsto V(J)$ induce $S O_{2 m^{-}}$ equivariant diffeomorphisms

$$
\mathbf{P}\left(P \mathbf{S}^{+}\right) \stackrel{K}{\mapsto} \mathcal{I}_{2 m}^{+} \stackrel{V}{\mapsto} \mathcal{C}_{m}^{+} \quad \text { and } \quad \mathbf{P}\left(P \boldsymbol{S}^{-}\right) \stackrel{K}{\mapsto} \mathcal{I}_{2 m}^{-} \stackrel{V}{\mapsto} \mathcal{C}_{m}^{-}
$$

We refer to the original book [3] for details.

For the sake of further discussion, we will fix $V \in I_{2 m}^{+}$and let $J \in \mathcal{C}_{m}^{+}$be the associated complex structure. Choose a unitary basis $\left\{e_{1}, J e_{1}, \ldots, e_{m}, J e_{m}\right\}$ of $\mathbf{R}^{2 m}$ and set

$$
\varepsilon_{j}=\frac{1}{\sqrt{2}}\left(e_{j}-i J e_{j}\right) \quad \bar{\varepsilon}_{j}=\frac{1}{\sqrt{2}}\left(e_{j}+i J e_{j}\right) .
$$

Define

$$
\omega_{j}=-\varepsilon_{j} \bar{\varepsilon}_{j} \quad \bar{\omega}_{j}=-\bar{\varepsilon}_{j} \varepsilon_{j} .
$$

Let $W$ be a linear subspace invariant under multiplication by $e_{j}$ and $J e_{j}$. Then there is a hermitian orthogonal direct sum decomposition

$$
W=W_{j} \oplus W_{j}^{\prime},
$$

where

$$
W_{j}=\bar{\omega}_{j} \cdot W=\operatorname{ker}\left(\left.\mu_{\bar{\varepsilon}_{j}}\right|_{W}\right) \text { and } \quad W_{j}=\omega_{j} \cdot W=\operatorname{ker}\left(\left.\mu_{\varepsilon_{j}}\right|_{W}\right)
$$

and, where $\mu_{\varepsilon_{j}}: W \rightarrow W$ is defined by $\mu_{\varepsilon_{j}}(w)=\varepsilon_{j} \cdot w$. By direct inductive calculation, we can construct

$$
\boldsymbol{S}_{m}=\operatorname{ker}\left(\mu_{\bar{\varepsilon}_{1}}\right) \cap \cdots \cap \operatorname{ker}\left(\mu_{\bar{\varepsilon}_{m}}\right) \quad \operatorname{dim}_{\mathbf{C}} \boldsymbol{S}_{m}=1
$$

The complex volume form $\omega_{\mathbf{C}}=i^{m} e_{1} J e_{1} \cdots e_{m} J e_{m}$ has the value +1 on $\$_{m}$ because $\bar{\varepsilon}_{j} \sigma=0 \Rightarrow-i e_{j} J e_{j} \sigma=\sigma$. Therefore, $\boldsymbol{\$}_{m} \subset \mathbf{S}_{\mathbf{C}}^{+}$.

We clearly have that $V(J)=\operatorname{ker} j_{\sigma}$ for $\sigma \in \boldsymbol{S}_{m}$. Hence $\boldsymbol{S}_{m}$ is independent of the choice of unitary basis and the map $V \mapsto\left[\mathbf{S}_{m}\right]$ gives the desired map $K^{-1}$ for the above proposition.

Definition 2.5. The bundle $\tau(X) \cong \mathbf{P}\left(P \mathbf{S}^{+}\right)$is called the twistor space of $X$. 
Note that $\mathbf{P}\left(P \$^{+}\right)$is an $\mathrm{SO}_{2 m}$-bundle and is globally defined whether or not $X$ is a spin manifold.

The total space of $\tau(X)$ carries a canonical almost complex structure defined by using the canonical decomposition of tangent space of $\tau(X)$, which is induced by the Riemannian connection of $X$.

$$
T(\tau(X))=\mathcal{V} \oplus \mathcal{H},
$$

where $\mathcal{H}$ is a field of horizontal planes and $\mathcal{V}$ is the field of tangent planes to the fibers. As noted, $\mathcal{V}$ has an almost complex structure integrable on the fibers since the fiber is naturally homogeneous complex manifold $\left(\cong S O_{2 m} / U(m)\right)$. The bundle $\mathcal{H}$ has a "tautological" almost complex structure defined, via the identification $\pi_{*}: \mathcal{H}_{J} \rightarrow T X$, to be the structure $d$ itself.

The question of integrability of $J$ already accomplished by M. Michelsohn.

Theorem 2.6. $[3,4]$ Let $X$ be an oriented (even-dimensional) riemannian manifold with an almost complex structure determined by a projective spinor field $u \in \Gamma(\tau(X))$. Then this almost complex structure is integrable if and only if $u$ is holomorphic.

This will be proved in Remark 3.3. As mentioned above, $\tau(X)$ carries a canonical almost complex structure. Now a $C^{1}$-map between almost complex manifolds $f:\left(X, J_{X}\right) \rightarrow\left(Y, J_{Y}\right)$ will be called holomorphic (resp. anti-holomorphic) if its differential $f_{*}$ is everywhere $J$-linear(resp. anti- $J$-linear) i.e., if $f_{*} \circ J_{X}= \pm J_{Y} \circ f_{*}$ respectively.

REMARK 2.7. More succinctly one could say that cross-section of $\tau(X)$ induce almost complex structure, and holomorphic cross-section induce the integrable ones However, the condition that a cross-section $u$ be holomorphic is not linear since the complex structure on $X$ depends itself on $u$.

We will prove that the complimentary condition for the holomorphicity, which is anti-holomorphic and harmonic is equivalent to that $u$ induce a symplectic structure on $X$.

Definition 2.8. $\omega \in \Omega^{2}(X)$ is a symplectic form if it is non-degenerate closed form. Moreover, $(X, \omega)$ is called a symplectic structure on $X$.

Given a twistor $u \in \mathbf{P}(P \boldsymbol{\$})$, there is naturally associated non-degenerate differential 2-form. It is induced by the hermitian metric with 
respect to the almost complex structure $J$ and Riemannian metric $g$ on $T X$ i.e.,

$$
\omega(v, w) \equiv g(J v, w),
$$

where $J$ is the almost complex structure corresponding to $s \in \mathbf{P}(P \$)$. Moreover it can be written as in terms of unitary basis, in other words, $\omega=\sum_{i=0}^{m} e_{i}^{*} \wedge\left(J e_{i}\right)^{*}$, where $e^{*} \in T^{*} X$ such that $e^{*}(v)=g(e, v) \in \mathbf{R}$. Recall that $\omega_{j}=-\varepsilon_{j} \bar{\varepsilon}_{j}$ for complex unitary basis $\left\{\varepsilon_{1}, \ldots, \varepsilon_{m}, \bar{\varepsilon}_{1} \cdots \bar{\varepsilon}_{m}\right\}$ of $(T X \otimes \mathbf{C})$. Since $\omega_{j}=-\varepsilon_{j} \bar{\varepsilon}_{j}=1-i e_{j} \cdot J e_{j}, i \omega=m-\sum_{j} \omega_{j}$.

$$
\begin{aligned}
\omega_{1} \cdots \omega_{m}= & \prod\left(1-i e_{j} \cdot J e_{j}\right) \\
= & 1-i \sum e_{j} \cdot J e_{j}-\sum_{j \neq k}\left(e_{j} \cdot J e_{j}\right) \cdot\left(e_{k} \cdot J e_{k}\right)+\cdots \\
= & 1-i \omega+(1 / 2)(-1)^{2} i \omega \wedge i \omega+\cdots \\
& +(1 / m !)(-1)^{m} i \omega \wedge \cdots i \omega \\
= & 1-i \omega+(1 / 2 !)(-i)^{2} \omega^{2}+\cdots+(1 / m !)(-i)^{m} \omega^{m},
\end{aligned}
$$

where $\omega^{k}=\overbrace{\omega \wedge \cdots \wedge \omega}^{k \text { times }} \in \Omega^{2 k}(X)$.

REMARK 2.9. The above equality comes from the identification between $T X$ and $T X^{*}$ via Riemannian metric. Note that $(1 / m !) i^{m} \omega^{m}=$ $i^{m} e_{1} \cdot J e_{1} \cdots e_{m} \cdot J e_{m}=\omega_{\mathbf{C}}$.

Note that ${ }^{*} \mathbf{C} \omega^{k}=k ! /(m-k) ! \omega^{m-k}$ i.e.,

$$
d \omega=0 \Leftrightarrow d \omega=d^{*} \omega=0 \Leftrightarrow \triangle_{g}\left(\omega_{1}+\cdots+\omega_{m}\right)=0,
$$

where $\triangle_{g}$ is the Laplacian operator with respect to metric $g$. Hence we have that $\omega$ defines a symplectic form if and only if $\tilde{\omega}=\omega_{1}+\cdots+\omega_{m}$ is harmonic. Our goal is to prove the following theorem.

THEoREM 2.10. Let $X$ be an oriented (even-dimensional) riemannian manifold with an almost complex structure determined by a projective spinor field $u \in \Gamma(\tau(X))$. Then this almost complex structure carries symplectic structure if and only if $u$ is harmonic and anti-holomorphic.

The product element, $q=\bar{\omega}_{1} \cdots \bar{\omega}_{m}$ (conjugate of the above product), of the complexified Clifford algebra $\mathrm{Cl}_{2 m}(X)$ can be characterized at least locally by an element of $q \in \operatorname{End}\left(\mathbf{S}^{+}\right)$such that

$$
q(\sigma)=\left\{\begin{array}{clll}
0 & & \text { if } & \sigma \in s^{\perp} \subset \mathbf{S}_{\mathbf{C}} \\
k \sigma & k \in \mathbf{C}^{*} & \text { and if } & {[\sigma]=s}
\end{array}\right.
$$


Note that we have not defined a complex spin representation $\boldsymbol{S}_{\mathbf{C}}$ globally over $X$. Without any specification of the complex spinor bundle, the $\bar{\omega}_{1} \cdots \bar{\omega}_{m}$ is well-defined as an element of $\mathbf{C l}_{2 m}(X)$. Using the almost complex structure associated with the twistor $u$, we can define canonical $\operatorname{spin}^{c}$ structure and canonical complex spin representation. Given the canonical Spin ${ }^{c}$ representation, the product element $q=2^{m} u \otimes u^{*}$, which is an element of $q \in \operatorname{End}_{\mathbf{C}}\left(\boldsymbol{S}^{+}\right)$in a way of that $q(\alpha)=\langle\alpha, u\rangle u$. In the next section, we will prove that $(d \omega) u=0$ if and only if $\mathbf{D} u=0$ by using the action of $q$.

\section{3. $\mathrm{Spin}^{c}$ representation and proof of Theorem 2.10}

Since $\operatorname{Spin}_{n}^{c} \equiv \operatorname{Spin}_{n} \times \mathbf{z}_{2} U(1)$, we have a short exact sequence

$$
0 \longrightarrow \mathbf{Z}_{2} \longrightarrow \operatorname{Spin}_{n}^{c} \stackrel{\xi}{\longrightarrow} S O_{n} \times U(1) \longrightarrow 1 .
$$

A principal $S O_{n}$-bundle $P$ carries a $\operatorname{Spin}^{C}$ structure if any only if the $w_{2}(P)$ is the mod 2 reduction of an integral class. Given a twistor $u \in \mathbf{P}\left(P \mathbf{S}^{+}\right)$, there is the canonical orthogonal almost complex structure $J$ on $T X$ asso siated with $u$. This $J$ defines a canonical $\operatorname{Spin}^{c}$ structure $\operatorname{det}_{\mathbf{C}} T X=K_{X}^{-1}$ since the first Chern class of $K_{X}^{-1}$ is an integral lift of the second Stiefel Whitney class, i.e., $c_{1}\left(K_{X}^{-1}\right) \equiv w_{2}(X) \bmod 2$. Let $\$_{\mathrm{C}}$ be the associated spinor bundle. Using the complex volume form $\sqrt{-1}^{m} e_{1} \cdot J e_{1} \cdots e_{m} \cdots J e_{m}$, we have the decomposition of $\$_{\mathbf{C}}$ by the \pm -eigenspace of the complex volume element, where $\boldsymbol{\$}^{ \pm}=\left(1 \pm \omega_{\mathbf{C}}\right) \boldsymbol{\$}_{\mathbf{C}}$. Let

$$
\varepsilon_{j}=\frac{1}{\sqrt{2}}\left(e_{j}-i J e_{j}\right) \quad \text { and } \quad \bar{\varepsilon}_{j}=\frac{1}{\sqrt{2}}\left(e_{j}+i J e_{j}\right)
$$

be an unitary basis for $T X$ as above. Define

$$
\$_{\mathbf{C}} \cong \oplus \boldsymbol{S}_{i_{1}, \ldots, i_{m}} \cong \oplus \operatorname{ker}\left(\mu_{\varepsilon_{i_{1}}}\right) \cap \cdots \operatorname{ker}\left(\mu_{\varepsilon_{i_{m}}}\right)
$$

where $\mu_{\varepsilon_{i_{k}}}=\left\{\begin{array}{ll}\mu_{\varepsilon_{k}} & i_{k}=k \\ \mu_{\overline{\varepsilon_{\bar{k}}}} & i_{k}=\bar{k}\end{array}\right.$. Let $\sigma=\left\{i_{1}, \ldots, i_{m}\right\}$ be the complex index used as above, define $|\sigma|$ be the number of elements of the subset $\left\{i_{k}=k\right\}$. Then we have

$$
\mathbf{S}_{\mathbf{C}}^{+} \cong \bigoplus_{|\sigma|=2 i} \mathbf{S}_{i_{1} \cdots i_{m}} \quad \text { and } \quad \mathbf{S}_{\mathbf{C}}^{-} \cong \bigoplus_{|\sigma|=2 i-1} \mathbf{S}_{i_{1} \cdots i_{m}}
$$

Especially, the twistor $u$ is contained in $\boldsymbol{\$}_{\overline{1}, \ldots, \bar{m}}$ which is characterized as $\bar{\varepsilon}_{j} \cdot u=0$ for all $j$. We can express the Dirac operator in terms of the 
unitary basis, which follows that

$$
\begin{aligned}
\not D & =e_{j} \cdot \nabla_{e_{j}}+J e_{j} \cdot \nabla_{J e_{j}} \\
& =\frac{1}{2}\left(\varepsilon_{j}+\bar{\varepsilon}_{j}\right) \cdot \nabla_{\varepsilon_{j}+\bar{\varepsilon}_{j}}-\frac{1}{2}\left(\varepsilon_{j}-\bar{\varepsilon}_{j}\right) \cdot \nabla_{\varepsilon_{j}-\bar{\varepsilon}_{j}} \\
& =\bar{\varepsilon}_{j} \cdot \nabla_{\varepsilon_{j}}+\varepsilon_{j} \cdot \nabla_{\bar{\varepsilon}_{j}} .
\end{aligned}
$$

REMARK 3.1. Note that the covariant derivative $\nabla$ is $\operatorname{Spin}^{c}$ connection which is induced from both the Levi-Civita connection and the $U(1)$ connection on $K_{X}^{-1}$ It should be well-noticed that our theorem is nothing to do with a $U(1)$ connection. Even though the condition we have imposed is related to simply "local" question, the spin ${ }^{c}$ structure enable us to work with globally. Furthermore, the following argument we will present below works finely without any $\operatorname{spin}^{c}$ structure.

To define a Dirac operator on the spinors, we should specify a $U(1)$ connection on $K_{X}^{-1}$. There is a canonical $U(1)$ connection unique up to gauge transformation $A_{0}$ such that $\langle\nabla u, u\rangle=0$. We will abuse the notation $\rrbracket$ for the Dirac operator, $\Phi_{A_{0}}$, which is induced by the Levi-Civita connection and the canonical $U(1)$ connection $A_{0}$. Our index notation convention indicates that $\nabla_{\tilde{e}_{j}} \tilde{e}_{k}=\sum_{l} \tilde{\omega}_{k}^{l}\left(\tilde{e}_{j}\right) e_{l}$ and $\Gamma_{j, k}^{l}=\tilde{\omega}_{k}^{l}\left(\tilde{e}_{j}\right)$ is the Chistoffel symbol. If $e_{j}=\tilde{e}_{2 j-1}, J e_{j}=\tilde{e}_{2 j}$ then $\varepsilon_{j}=\frac{1}{\sqrt{2}}\left(\tilde{e}_{2 j-1}-i \tilde{e}_{2 j}\right)$, where $i=\sqrt{-1}$. Then we have

$$
\begin{array}{ll}
\nabla_{\varepsilon_{j}} \bar{\varepsilon}_{k}=a_{j, k}^{l} \varepsilon_{l}+c_{j, k}^{l} \bar{\varepsilon}_{l}, & \nabla_{\bar{\varepsilon}_{j}} \varepsilon_{k}=\bar{a}_{j, k}^{l} \bar{\varepsilon}_{l}+\bar{c}_{j, k}^{l} \varepsilon_{l} \\
\nabla_{\bar{\varepsilon}_{j}} \bar{\varepsilon}_{k}=b_{j, k}^{l} \varepsilon_{l}+d_{j, k}^{l} \bar{\varepsilon}_{l}, & \nabla_{\varepsilon_{j}} \varepsilon_{k}=\bar{b}_{j, k}^{l} \bar{\varepsilon}_{l}+\bar{d}_{j, k}^{l} \varepsilon_{l} .
\end{array}
$$

Since the Levi-Civita connection is naturally compatible with the Hermitian metric on $T X \otimes \mathbf{C}$, we have

$$
a_{j, k}^{l}=\left\langle\nabla_{\varepsilon_{j}} \bar{\varepsilon}_{k}, \varepsilon_{l}\right\rangle=-\left\langle\bar{\varepsilon}_{k}, \nabla_{\bar{\varepsilon}_{j}} \varepsilon_{l}\right\rangle=-a_{j, l}^{k} \text {. }
$$

By the same manner, we have

$$
b_{j, k}^{l}=-b_{j, l}^{k} \quad \text { and } \quad c_{j, k}^{l}=-\bar{d}_{j, l}^{k} .
$$

LEMMA 3.2. Let $u$ be a section of twistor space and $J$ be the associated orthogonal almost complex structure. Then $u$ is anti-holomorphic if and only if $a_{j, k}^{l}=0$ for all $j, k, l$ and $u$ is holomorphic section if and only $b_{j, k}^{l}=0$ for all $j, k, l$. 
First of all, we have to find the covariant derivative of $u$ which is

$$
\nabla u=\frac{1}{2} \sum_{k<l} \tilde{\omega}_{k}^{l} \otimes \tilde{e}_{l} \tilde{e}_{k} \cdot u
$$

where $\tilde{\omega}$ is the $s o(2 m)$ connection 1-form (Levi-Civita connection with respect to $g$ ) $\varepsilon s s o c i a t e d$ with orthonormal basis $\left\{\tilde{e}_{1}, \ldots, \tilde{e}_{2 m}\right\}$. Let

$$
\nabla_{\varepsilon_{j}} u \equiv \frac{1}{2} \sum_{k<l} \tilde{a}_{j, k}^{l} \otimes \varepsilon_{l} \cdot \varepsilon_{k} \cdot u \bmod \langle u\rangle .
$$

The coefficient, $\tilde{a}_{j, k}^{l}$ can be derived as follows:

$$
\bar{\varepsilon}_{t} \cdot u=0 \quad \text { for all } t .
$$

By taking covariant derivative $\nabla_{\varepsilon_{j}}$, we have

$$
\left(\nabla_{\varepsilon_{j}} \bar{\varepsilon}_{t}\right) \cdot u+\bar{\varepsilon}_{t} \cdot \nabla_{\varepsilon_{j}} u=0 .
$$

Hence

$$
\begin{aligned}
\left(\nabla_{\varepsilon_{j}} \bar{\varepsilon}_{t}\right) \cdot u & =-\bar{\varepsilon}_{t} \cdot \sum_{k<l} \frac{1}{2} \tilde{a}_{j, k}^{l} \varepsilon_{l} \varepsilon_{k} \cdot u \\
& =-\sum \frac{1}{2} \tilde{a}_{j, k}^{l} \bar{\varepsilon}_{t} \varepsilon_{l} \varepsilon_{k} \cdot u \\
& =\left\{\begin{array}{cl}
\tilde{a}_{j, k}^{l} \varepsilon_{l} \cdot u & \text { for } k=t<l \\
-\tilde{a}_{j, k}^{t} \varepsilon_{k} \cdot u & \text { for } l=t>k .
\end{array}\right.
\end{aligned}
$$

Since $\bar{\omega}_{j}=-\bar{\varepsilon}_{j} \cdot \varepsilon_{j} \cdot u=2 u$. Therefore we have

$$
\left\langle\nabla_{\varepsilon_{j}} \bar{\varepsilon}_{t}, \varepsilon_{s}\right\rangle=a_{j, t}^{s}=\tilde{a}_{j, t}^{s} .
$$

We get $\tilde{a}_{j, k}^{l}=\left\langle\nabla_{\varepsilon_{j}} \varepsilon_{k}, \varepsilon_{l}\right\rangle$. By the analogous method, we can get

$$
\nabla_{\bar{\varepsilon}_{j}} u \equiv \frac{1}{2} \sum_{k<l} b_{j, k}^{l} \otimes \varepsilon_{l} \cdot \varepsilon_{k} \cdot u \bmod \langle u\rangle .
$$

With this understood, it can be rephrased that $u$ is anti-holomorphic $\Leftrightarrow \bar{\varepsilon}_{t} \cdot \nabla_{\varepsilon_{j}} u=0 \Leftrightarrow a_{j, k}^{l}=0 \Leftrightarrow\left\langle\nabla_{\varepsilon_{j}} \bar{\varepsilon}_{k}, \varepsilon_{l}\right\rangle=0$ for all $j, k, l$. Also $u$ is holomorphic $\Leftrightarrow \overline{\varepsilon_{t}} \cdot \nabla_{\bar{\varepsilon}_{j}} u=0 \Leftrightarrow a_{j, k}^{l}=0 \Leftrightarrow\left\langle\nabla_{\varepsilon_{j}} \bar{\varepsilon}_{k}, \varepsilon_{l}\right\rangle=0$ for all $j, k, l$.

REMARK 3.3. From the torsion free condition of Levi-Civita connection, we have

$$
b_{j, k}^{l}-b_{k, j}^{l}=\left\langle\nabla_{\bar{\varepsilon}_{j}} \bar{\varepsilon}_{k}-\nabla_{\bar{\varepsilon}_{k}} \bar{\varepsilon}_{j}, \varepsilon_{l}\right\rangle=\left\langle\left[\bar{\varepsilon}_{j}, \bar{\varepsilon}_{k}\right], \varepsilon_{l}\right\rangle .
$$

Since the anti-commutativity between upper index and right lower index $b_{j, k}^{l}=-b_{j, l}^{k}$, we can get an equivalent condition which says that $b_{j, k}^{l}-$ 
$b_{k, j}^{l}=0$ if and only if $b_{j, k}^{l}=0$. Hence it is easy to prove the Theorem 2.6 from the above equation.

We want to find an equivalent condition for the harmonic two form $\omega$ i.e., $\mathbb{D} \omega=\left(d+d^{*}\right) \omega=0$, where $d^{*}$ is the formal adjoint of $d$ with respect to $g$. The following lemma is about it.

Proposition 3.4. Let $\omega=-m+\sum_{k} \omega_{k}$ be the purely imaginary part of the Hermitian metric. Then $\mathbf{D} \omega=0$ if and only if $a_{j, k}^{l}=0$ and $b_{j, k}^{l}+b_{l, j}^{k}+b_{k, l}^{j}=0$ for all $j, k, l$.

Proof. Since $\omega$ is purely imaginary two form, we have

$$
\begin{aligned}
\not \supset \omega & =\sum_{j} \varepsilon_{j} \nabla_{\bar{\varepsilon}_{j}} \omega+\bar{\varepsilon}_{j} \nabla \varepsilon_{j} \omega \\
& =\sum_{j} \varepsilon_{j} \nabla_{\bar{\varepsilon}_{j}} \omega-\overline{\sum_{j} \varepsilon_{j} \nabla_{\bar{\varepsilon}_{j}} \omega} \\
& =2 i \operatorname{Im} \mathbb{D}^{\frac{1}{2}} \omega
\end{aligned}
$$

It suffices to consider the half part of the Dirac operator, it reads

$$
\begin{aligned}
-\mathbb{D}^{\frac{1}{2}} \omega & =\sum_{j}-\varepsilon_{j} \cdot \nabla_{\bar{\varepsilon}_{j}} \omega \\
& =\sum_{j, k}\left(\varepsilon_{j} \cdot\left(\nabla_{\bar{\varepsilon}_{j}} \varepsilon_{k}\right) \cdot \bar{\varepsilon}_{k}+\varepsilon_{j} \cdot \varepsilon_{k} \cdot \nabla_{\bar{\varepsilon}_{j}} \bar{\varepsilon}_{k}\right) \\
& =\sum_{j, k, l}\left(\bar{a}_{j, k}^{l} \varepsilon_{j} \bar{\varepsilon}_{l} \bar{\varepsilon}_{k}+\bar{c}_{j, k}^{l} \varepsilon_{j} \varepsilon_{l} \bar{\varepsilon}_{k}+b_{j, k}^{l} \varepsilon_{j} \varepsilon_{k} \varepsilon_{l}+d_{j, k}^{l} \varepsilon_{j} \varepsilon_{k} \bar{\varepsilon}_{k}\right) \\
& =\sum_{j, k, l}\left(\bar{a}_{j, k}^{l} \varepsilon_{j} \bar{\varepsilon}_{l} \bar{\varepsilon}_{k}+b_{j, k}^{l} \varepsilon_{j} \varepsilon_{k} \varepsilon_{l}\right)+\sum_{j, k, l}\left(\bar{c}_{j, l}^{k}+d_{j, k}^{l}\right) \varepsilon_{j} \varepsilon_{k} \bar{\varepsilon}_{l} \\
& =\sum_{i, j, l}\left(\bar{a}_{j, k}^{l} \varepsilon_{j} \bar{\varepsilon}_{l} \bar{\varepsilon}_{k}+b_{j, k}^{l} \varepsilon_{j} \varepsilon_{k} \varepsilon_{l}\right) .
\end{aligned}
$$

Hence $\not D \omega=0$ if and only if $\mathbb{D}^{\frac{1}{2}} \omega=0 \Leftrightarrow a_{j, k}^{l}=0$ for all $i, j, k$ and $\sum_{\sigma} b_{\sigma(j), \sigma(k)}^{\sigma(l)}$, where $\sigma$ is the permutation of $i, j, k$. The relation $b_{j, k}^{l}=$ $-b_{j, l}^{k}$ completes the proposition. 
Proof of Theorem 2.10. Since $\langle\nabla u, u\rangle=0$, we have

$$
\begin{aligned}
\not D u & =\sum_{j}\left(\bar{\varepsilon}_{j} \nabla_{\varepsilon_{j}} u+\varepsilon_{j} \nabla_{\bar{\varepsilon}_{j}} u\right) \\
& =\sum_{j, k, l}\left(\frac{1}{4} a_{j, k}^{l} \bar{\varepsilon}_{j} \varepsilon_{k} \varepsilon_{l} \cdot u+\frac{1}{4} b_{j, k}^{l} \varepsilon_{j} \varepsilon_{k} \varepsilon_{l} \cdot u\right) \\
& =\sum_{j, k} a_{j, j}^{k} \varepsilon_{k} \cdot u+\sum_{j<k<l} \frac{1}{2}\left(b_{j, k}^{l}+b_{l, j}{ }^{k}+b_{k, l}^{j}\right) \varepsilon_{j} \varepsilon_{k} \varepsilon_{l} \cdot u .
\end{aligned}
$$

Hence $u$ is anti-holomorphic pure spinor $\left(\bar{\varepsilon}_{t} \nabla_{\varepsilon_{j}}=0\right.$ for all $\left.t, j\right)$ and harmonic $(\mathbb{D} u=0)$ gives an equivalent condition for $\omega$ being a symplectic form. Note that given symplectic manifold $(X, \omega)$ has such a anti-holomorphic and harmonic twistor $u$ by choosing any almost complex structure which calibrate $\omega$.

Corollary 3.5. $(d \omega) \cdot u=0$ if and only if $u$ is harmonic, i.e., $\mathbf{D} u=0$.

Proof. Let $q=\prod_{j=1}^{m} \overline{\omega_{j}}=\prod_{j}\left(1+i e_{j} \cdot J e_{j}\right)$. Using the action $q$ on $u$, $q \cdot u=2^{m} u$, and taking Dirac operator on the both side, we can have

$$
\begin{aligned}
\not \mathbf{D} q \cdot u & =(\not \mathbf{D} q) \cdot u+\sum \tilde{e}_{j} q \cdot \nabla_{\tilde{e}_{j}} u \\
& =\not \mathbf{D} q \cdot u \quad \Leftarrow\left(\langle\nabla u, u\rangle=0 \Rightarrow q \cdot \nabla_{\tilde{e}_{j}} u=0\right) \\
& =\not \mathbf{D} u .
\end{aligned}
$$

Thus $\nsupseteq u=0$ if and only if $(\not D q) \cdot u=0$. Moreover since $(3 i)^{m}(-1)^{\frac{1}{2} p(p+1)}$ $\varphi \omega_{\mathbf{C}}=* \varphi$ for $\varphi \in \Omega^{p}(X)$ and $1 / k ! * \omega^{k}=1 /(m-k) ! \omega^{m-k}$, we have

$$
\begin{aligned}
\not D q= & \left(d+a^{*}\right) q=i d \omega+i^{2} / 2 ! d \omega^{2}+\cdots+i^{m-1} /(m-1) ! d \omega^{m-1} \\
& -i /(m-1) ! * d \omega^{m-1}-i^{2} /(m-2) ! * d \omega^{m-2}-\cdots-i^{m-1} * d \omega \\
= & i d \omega+i^{2} / 2 ! d \omega^{2}+\cdots+i^{m-1} /(m-1) ! d \omega^{m-1} \\
& i^{4 m+1} d \omega \cdot \omega_{\mathbf{C}}+i^{4 m+2} / 2 ! d \omega^{2} \cdot \omega_{\mathbf{C}}+\cdots \\
& +i^{m-1} /(m-1) ! d \omega^{m-1} \cdot \omega_{\mathbf{C}} .
\end{aligned}
$$

Since $\omega_{\mathbf{C}} u=u, \omega \cdot u=-(m i) u$, we have $d \omega^{k} \cdot u=k(d \omega) \wedge \omega^{k-1} u=$ $k(-m i)^{k-1} d \omega \cdot u$. Thus

$$
(\not D q) \cdot u=2 i\left(1+m+m^{2} / 2 !+\cdots+m^{m-2} /(m-2) !\right) d \omega \cdot u .
$$

This completes the proof.

REMARK 3.6. In dimension $2 m \leq 6$ every non-zero positive (or negative) spinor is pure, i.e., $P \boldsymbol{S}^{ \pm}=\boldsymbol{S}_{\mathrm{C}}^{ \pm}-0$. This is simply because the group $\mathrm{Spin}_{2 m}$ acts transitively on the unit sphere in $\boldsymbol{S}_{\mathrm{C}}^{ \pm}$in these dimensions. 
In dimension 4 , since $\varphi \in \Omega^{3}(X, \mathbf{R})$ acts on $u$ injectively, we get $(d \omega) \cdot u=0$ if and only if $d \omega=0$. Hence the harmonic spinor $u$, equivalently, anti-holomorphic twistor, gives a sufficient condition to induce a symplectic structure. The next corollary follows from it.

Corollary 3.7. In dimension 4 , Let $u$ be a nowhere vanishing section of positive complex spinor bundle. Suppose $\mathbb{D} u=0$ and $\langle\nabla u, u\rangle=$ 0 , then $X$ is symplectic 4-manifold.

Finally, suppose $\nabla u=0$. Then $u$ is then both holomorphic and antiholomorphic twistor. We have following corollary, which is proposition 9.8 in [3].

Corollary 3.8. If $u$ is parallel, then $(X, g, J)$ becomes a Kähler manifold.

\section{References}

[1] S. K. Donaldson, The Seiberg-Witten equations and 4-manifold topology, Bull. Amer. Math. Soc. (N. S.) 33 (1996), no. 1, 45-70.

[2] R. E. Gompf, A new construction of symplectic manifolds, Ann. of Math. (2) 142 (1995), no. 3, 527-595

[3] H. Blaine, Jr. Lawson and M.-L. Michelsohn, Spin geometry, Princeton Mathematical Series volume 38, Princeton University Press, Princeton, NJ, 1989.

[4] J. W. Morgan, The Seiberg-Witten equations and applications to the topology of smooth four-manifolds, Mathematical Notes volume 44, Princeton University Press, Princeton, NJ, 1996.

[5] C. H. Taubes, The Seiberg-Witten invariants and symplectic forms, Math. Res. Lett. 1 (1994), no. 6, 809-822.

[6] _ More constraints on symplectic forms from Seiberg-Witten invariants, Math. Res. Lett. 2 (1995), no. 1, 9-13.

Department of Mathematics Education

Konkuk University

Seoul 143-701, Korea

E-mail: dosjoe@konkuk.ac.kr 